\title{
Direction Trees
}

\author{
Robert E. Jamison \\ Mathematical Sciences Department, Clemson University, Clemson, SC 29634-1907, USA \\ Communicated by Jacob E. Goodman
}

\begin{abstract}
Given a finite set of noncollinear points in the real plane, it is shown that they can be connected to form a tree using lines, no two of which are parallel.
\end{abstract}

Let $X$ be a finite set of points in the Euclidean plane. A direction tree for $X$ is a tree $T$ whose nodes are the points of $X$ such that any two edges of $T$ determine lines in different directions. Some direction trees are shown in Fig. 1. An obvious necessary condition for a set $X$ of $n$ points to possess a direction tree is that the set of lines connecting pairs of points of $X$ should determine at least $n-1$ different directions. This was conjectured to be the case for all noncollinear $X$ by Scott [6] and proved by Ungar (Theorem 2 of [7]) using the allowable sequences of Goodman and Pollack [1].
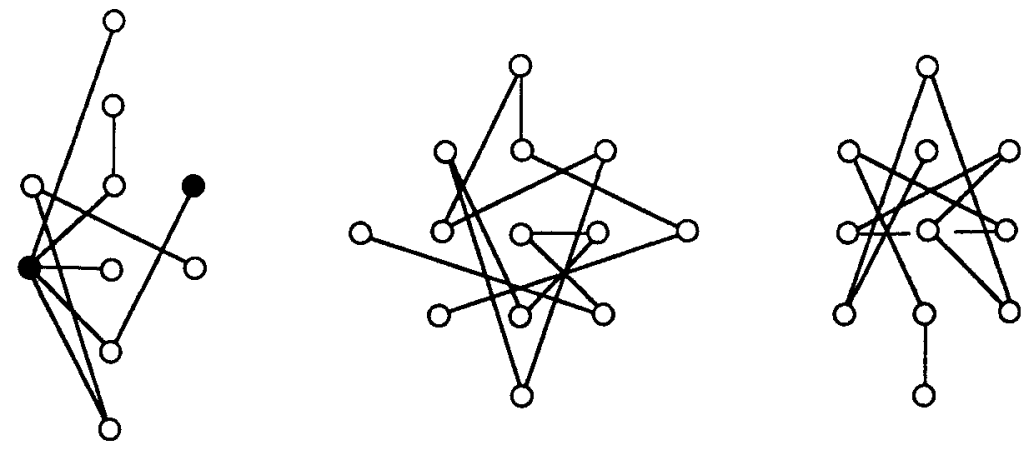

Fig. 1. Some direction trees. 
Ungar's Theorem [7]. Any n noncollinear points in the real affine plane determine at least $n-1$ directions of connecting lines. Moreover, equality is possible only for $n$ odd.

The goal here is to derive the existence of direction trees from Ungar's theorem using Hall's celebrated theorem [2] on distinct representatives.

In the ensuing development, it is convenient to think of the complete graph on $X$ with its edges partitioned into parallel classes. Two edges $u-v$ and $x-y$ are parallel iff the corresponding lines $u v$ and $x y$ through their endpoints are parallel (or coincide). Equivalently, one can think of each edge as labeled by the slope of the line through its endpoints. (Vertical lines have slope $\infty$.) This edge-labeled complete graph is the direction pattern of $X$. A direction tree is then a spanning tree in the direction pattern, whose edges all bear different labels. It is in fact possible to prove the analogue of Theorem $A$ in the abstract setting of edge-labeled complete graphs which satisfy an analogue of Ungar's theorem [5].

For any finite set $X$ in the real affine plane, let

$|X|=$ the number of points in $X$,

$X(X)=$ the sets of directions (slopes) determined by the connecting lines of $X$,

$|\chi(X)|=$ the number of directions (slopes) determined by $X$, $\delta(X)=|\chi(X)|-|X|$.

By Ungar's theorem, $\delta(X) \geq-1$ if $X$ is noncollinear. We call $X$ critical if $X$ is noncollinear and $\delta(X)=-1$. (Thus one and two points are explicitly excluded from the class of critical sets.)

Suppose $Z \subseteq X$ and that $S$ is a direction tree for $Z$. A direction tree $T$ for $X$ strongly extends $S$ providing $S$ is a subtree of $T$ and no edge of $T$ not in $Z$ is parallel to any edge of $Z$. That is, in extending $S$, the tree $T$ uses only new directions not in $Z$.

Lemma 1. Suppose $Z \subseteq X$ and that $\delta(Z) \leq \delta(Y)$ for every $Y$ with $Z \subseteq Y \subseteq X$. Then any direction tree for $Z$ may be strongly extended to a direction tree for $X$.

Proof. Arbitrarily order the points of $X \backslash Z$ as $x_{1}, x_{2}, \ldots, x_{m}$. For each $i \geq 1$, set $Z_{i}=Z \cup\left\{x_{1}, x_{2}, \ldots, x_{i}\right\}$. Let $\Delta_{i}$ consist of all slopes which are not in $\chi(Z)$ but which do occur on an edge from $x_{i}$ to another point of $Z_{i}$. Now for any set $I$ of $k \leq m$ indices, consider the set $Y=Z \cup\left\{x_{i}: i \in I\right\}$. By hypothesis, $\delta(Y) \geq \delta(Z)$. This yields $|\chi(Y)| \geq|\chi(Z)|+k$ since $k=|Y|-|Z|$. Thus there are at least $k$ slopes among points in $Y$ that do not occur in $Z$.

Suppose $\sigma$ is such a slope. Since $\sigma$ does not occur in $Z, \sigma$ must be the slope of a line of $Y$ either of the form $x_{i} z$ where $i \in I$ and $z \in Z$ or of the form $x_{i} x_{j}$ where $i \in I, j \in I$, and $i>j$. In either case, it is clear that $\sigma$ belongs to $\Delta_{i}$. Thus the union of $\Delta_{i}$ for all $i$ in $I$ contains all slopes in $\chi(Y)$ that are not in $\chi(Z)$. As we have shown that there are at least $k=|I|$ of these, it follows that the $\Delta_{i}$ satisfy Hall's conditions for a system of distinct representatives [2]. 
Let $\sigma_{i} \in \Delta_{i}$ be a system of distinct representatives. Each $\sigma_{i}$ is the slope of an edge of the form $x_{i} y_{i}$ where either $y_{i}=x_{j}$ for some $j<i$ or $y_{i} \in Z$. The desired strong extension $T$ may now be obtained by adjoining to $S$ the edges $x_{i} y_{i}$ for each $i$ in $I$.

Lemma 2. If $K$ is a critical subset of $X$, then any direction tree for $K$ can be extended to a direction tree for all of $X$.

Proof. This follows at once from Ungar's theorem [7] and Lemma 1.

Lemma 3. If $K$ is critical, then any slope in $\chi(K)$ occurs on at least two edges of $K$.

Proof. Consider any point $x$ of $K$. Since $K$ is critical, $|K|$ is odd by Ungar's theorem. Hence $|K \backslash x|$ is even, so $K \backslash x$ is not critical. Thus $\delta(K \backslash x) \geq 0$. But since $\chi(K \backslash x) \subseteq \chi(K)$, we have

$$
0 \leq \delta(K \backslash x)=|\chi(K \backslash x)|-|K \backslash x| \leq|\chi(K)|-(|K|-1)=\delta(K)+1=0 .
$$

Hence $\delta(. K \backslash x)=0$ and $\chi(K)=\chi(K \backslash x)$.

Now consider any edge $x y$ of $K$. Since $\chi(K)=\chi(K \backslash x)$, there must be an edge in $K \backslash x$ with the same slope as $x y$. Hence the slope of $x y$ occurs on at least two edges of $K$.

Lemma 4. Suppose $Z \subseteq X$ and $\delta(Z)=0$. Suppose $x z$ is an edge of $Z$ which is not parallel to another edge of $Z$. Then any direction tree for $Z$ which does not use $x z$ may be extended to a direction tree for $X$.

Proof. Let $S$ be a direction tree for $Z$ that does not use $x z$. If $Z$ lies in no critical subset of $X$, then $\delta(Y) \geq 0=\delta(Z)$ for all $Y$ with $Z \subseteq Y \subseteq X$. Thus $S$ can be extended to $X$ by Lemma 1 .

Thus, suppose $Z$ lies in a critical subset of $X$. Let $K$ be a critical subset of $X$ of smallest cardinality which contains $Z$. Then $x z$ is parallel to some other edge $u v$ of $K$ by Lemma 3. Since the edge $u v$ is not in $Z$, one of $u$ or $v$ is not in $Z$, say $v \notin Z$. By minimal choice of $K$, for any $Y$ with $Z \subseteq Y \subseteq K \backslash v$, we have $\delta(Y) \geq 0$. Thus $S$ can be strongly extended to a direction tree $T^{\prime}$ for $K \backslash v$ by Lemma 1 . Thus $T^{\prime}$ uses no edge parallel to $x z$. Hence, adjoining the edge $u v$ to $T^{\prime}$ produces a direction tree $T^{\prime \prime}$ for $K$. By Lemma 2, $T^{\prime \prime}$ can be extended to all of $X$.

Theorem A. Every noncollinear (finite) set $X$ in the plane possesses a direction tree. Moreover, if $x, y$, and $z$ are noncollinear points of $X$, then the path $x-y-z$ can be extended to a direction tree for $X$.

Proof. Let $Z=\{x, y, z\}$. Then $\delta(Z)=0$ and $x z$ is parallel to no other edge of $Z$. Thus $x-y-z$ can be extended to a direction tree for $X$ by Lemma 4 . 

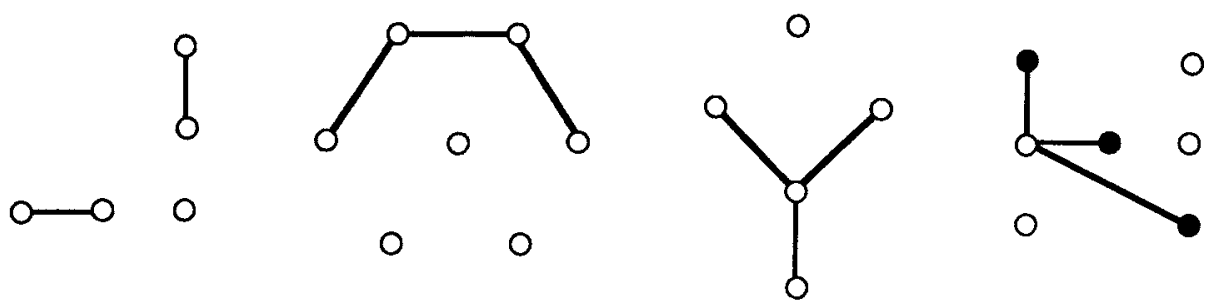

Fig. 2. False starts for direction trees.

By the above theorem, any two nonparallel but incident edges of any configuration $X$ can be extended to a direction tree for $X$. The first configuration in Fig. 2 shows that this is not necessarily the case if the two edges are not incident. It is tempting to conjecture that if every point of $X$ lies on at least three lines, then any two nonparallel edges can be extended to a direction tree.

The remaining configurations in Fig. 2 have three mutually nonparallel but connected edges which fail to extend to direction trees. In the last case, the configuration is critical, so any direction tree must use all the slopes. The slope of the main diagonal cannot be used, however, without creating a cycle with the given edges.

On the other hand, Lemmas 2 and 4 do give a lot of universally extendible direction trees-that is, direction trees which may be extended to a direction tree on any superset. There are four infinite families and 102 sporadic critical configurations known [3]. By Lemma 2 any direction tree on one of these configurations is universally extendible. Several types of universally extendible trees that arise from Lemma 4 are shown in Fig. 3. The trees in Fig. 1 are also universally extendible: the first by Lemma 4 and the last two by Lemma 2 .

By Lemma 1, if $\delta(X) \leq \delta(U)$ for every superset $U$ of $X$, then any direction tree on $X$ is universally extendible. Curiously, no $X$ which is not critical has as yet been shown to possess this property, but there are undoubtedly many noncritical such sets. Indeed, the set of vertices of the regular $(2 k+1)$-gon, for $k \geq 3$, is probably such a set.

We close with a brief discussion of three special types of direction trees.
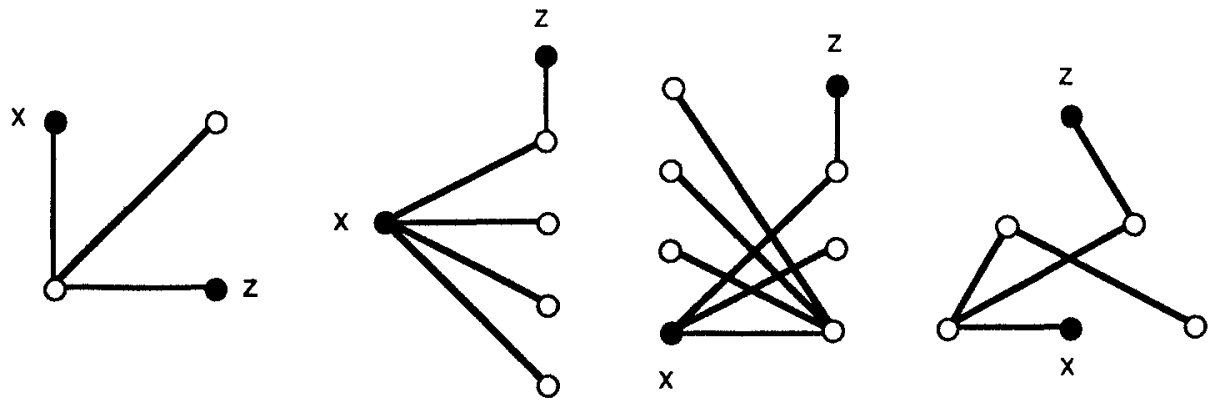

Fig. 3. Some universally extendible direction trees. 
In the definition of a "direction tree on a set $X$," an important distinction must be made between the edge $x y$ joining two points of $X$ and the line segment $[x, y]$ between $x$ and $y$. The edge $x y$ is regarded as joining only its two endpoints $x$ and $y$ even if the segment contains other points of $X$. An edge $x y$ of the direction pattern of $X$ is proper provided the line segment $[x, y]$ contains no points of $X$ except $x$ and $y$. The first two trees in Fig. 1 have only proper edges; the last tree has an improper horizontal edge. For graphical clarity in drawing direction trees, it is desirable to have only proper edges. As the result below shows, it is easy to obtain from any direction tree one with only proper edges.

Theorem B. Let $T$ be a direction tree on a set $X$. There exists a direction tree $S$ for $X$ such that:

(1) each edge of $S$ is proper,

(2) every proper edge of $T$ is an edge of $S$, and

(3) every edge of $S$ is parallel to an edge of $T$.

Proof. Among all direction trees for $X$ which satisfy (2) and (3), choose one, say $S$, with the least number of improper edges. Suppose $p q$ is an improper edge of $S$. Cutting the edge $p q$ of $S$ breaks $X$ into components, say $P$ and $Q$, containing $p$ and $q$, respectively. Along the segment $[p, q]$ from $p$ to $q$, let $p^{\prime}$ be the last point of $P$. The next point $q^{\prime}$ of $X$ on this segment is then in $Q$. Inserting the edge $p^{\prime} q^{\prime}$ reconnects $X$ into a tree $S^{\prime}$. Since $p^{\prime} q^{\prime}$ is an edge parallel to the deleted edge $p q$, it follows that $S^{\prime}$ is a direction tree also satisfying (2) and (3) but with one fewer improper edge than $S$. Hence by the minimal choice of $S$, it must satisfy (1) also.

A direction path is a direction tree that is a path. That is, a direction path for $X$ is a listing $x_{1}, x_{2}, \ldots, x_{n}$ of the points of $X$ so that the $n-1$ lines $x_{i} x_{+1}$ all have different slopes. The last two trees in Fig. 1 are direction paths. Since at most two consecutive points on a direction path can lie on the same line, if $X$ has a direction path, then no connecting line of $X$ can have more than $\frac{1}{2}|X|+1$ points. There can be, however, more subtle restrictions on the maximum size of connecting lines. Indeed, as shown in Theorem 5.2 of [4], there is an infinite class of configurations having no direction paths in which the largest connecting lines have only $\frac{1}{3}|X|+3$ points. The first configuration in Fig. 1 is the smallest member of this class. On the basis of the evidence in [4], it seems reasonable to conjecture that if no line of $X$ has more than $\frac{1}{3}|X|$ points, then $X$ has a direction path. A particularly appealing case of this conjecture remains (annoyingly) unresolved.

Conjecture. If $X$ is a finite set of points, no three collinear, then $X$ has a direction path.

It is also of interest to look at direction trees of small radius. Let $r(X)$ denote the minimum radius of all direction trees for $X$. Let $r(n)$ denote the maximum of $r(X)$ over all noncollinear $X$ with $|X|=n$. It would be of interest to study the behavior of $r(n)$ as $n$ goes to infinity. It is conceivable that $r(n)$ may be bounded. 


\section{References}

1. J. E. Goodman and R. Pollack, A combinatorial perspective on some problems in geometry, Congr. Numer. 32 (1981), 383-394.

2. P. Hall, On representatives of subsets, J. London Math. Soc. 10 (1935), 26-32.

3. R. E. Jamison and D. Hill, A catalogue of sporadic slope-critical configurations, Congr. Numer. 40 (1983), 101-125.

4. R. E. Jamison, Direction paths, in preparation.

5. R. E. Jamison, Polychrome spanning subtrees of edge-colored graphs, in preparation.

6. P. R. Scott, On the sets of directions determined by n-points, Amer. Math. Monthly 77 (1970), 502-505.

7. P. Ungar, $2 N$ noncollinear points determine at least $2 N$ directions, $J$. Combin. Theory Ser. A 33 (1982), 343-347.

Received July 29, 1985, and in revised form April 28, 1986. 